as it is fairly common for the temperature to take 10 to 14 days to settle with certain resistant organisms such as enterococci. Bacteriologically negative patients should be treated as if they had a resistant organism such as the enterococcus.

The most outstanding new development in the treatment of infective endocarditis is probably in the field of surgery. Acute rupture of a cusp nearly always affects the aortic valve and appears either in the active phase of the infection or soon after the completion of treatment. If the aortic valve is damaged in this way it should be replaced without delay, and even in the most desperate circumstances the results may be surprisingly good.

A two-year study of 102 consecutive cases of infective endocarditis from Uganda ${ }^{5}$ provides an interesting contrast to the findings in Britain. Fifty-seven cases were diagnosed on clinical presentation (group 1), with positive blood cultures in 33. Staphylococci and streptocci were isolated with equal frequency and Str. viridans was present in only onequarter of positive cultures. In 24 cases blood cultures were negative and diagnosis was based on the classical clinical features. All of these were treated with penicillin and streptomycin. The mortality rate of $19 \%$ in these patients (group 1) compares favourably with that recorded elsewhere. In 45 further cases (group 2) diagnosis was made only at necropsy. Most of these presented an acute course terminating in cardiac failure, frequently due to acute valvular destruction in the apparent absence of pre-existing valvular disease. Three-quarters of the clinical cases and nearly two-thirds of the necropsy cases were patients below 40 years of age.

Rheumatic heart disease was present in two-thirds of those in group 1 and in one-quarter there was "no previously suspected heart lesion." In this latter subgroup infection with Staph. aureus predominated. In group 2 rheumatic heart disease was present in less than one-third of cases, and more than half the hearts showed no underlying heart disease. A detailed study of 28 of these necropsy cases (21 males and seven females) encountered in a 12-month period 6 showed that the infection involved a previously normal valve in 16, the aortic valve being affected in 13 cases. The patients with this isolated aortic endocarditis were all men, usually in their third or fourth decade, and it is suggested that the initial site of infection may have been the genitourinary tract.

Infective endocarditis presents a complex problem in both temperate and tropical countries. The role of immunological and other factors in the patient is uncertain. When we consider the large number of people at risk who have dental extractions or other procedures without any antibiotic cover, the incidence of the disease must be remarkably small. In two-thirds of patients no precipitating cause is detectable. The host's immunological response is probably more important than the infection, but in our present state of ignorance it remains obligatory to give prophylactic antibiotics for those at risk.

\footnotetext{
${ }^{1}$ Hayward, G. W., British Medical Fournal, 1973, 2, 706, 764.

2 Angrist, A. A., Oka, M., and Naao, K., in Pathology Annual, vol. 2, ed. S. C. Sommers. London, Butterworths, 1967.

3 Cordeiro, A., Corta, H., and Laginha, F., American fournal of Cardiology, 1965, 16, 477

4 Cream, J. J., and Turk, J. L., Clinical Allergy, 1971, 1, 235.

5 Somers, K., Patel, A. K., Steiner, I., D'Arbela, P. G., and Hutt, M. S. R. British Heart fournal, 1972, 34, 1107.

- Steiner, I., Patel, A. K., Hutt, M. S. R., and Somers, K., British Heart Fournal, 1973, 35, 159.
}

\section{Costing the N.H.S.}

If the N.H.S. is to meet new demands the Service cannot bank on an open-cheque on the Treasury. It must look for extra resources by using more economically the existing allocations-but this is easier said than done. Despite the Grey Book's rhetoric about "accountability,"l it is not clear in what statisitical currency this exercise is to be carried out. Most doctors will agree about the inadequacy of the data now available for measuring what is happening in the N.H.S. and for allocating costs to particular activities or patient groups. But, the diagnosis of inadequacy having been made, there is little agreement about how best to set about treating this crucial problem of management.

It is possible, however, to distinguish at least two approaches. The first is represented in an article by Opit and Cross (Supplement, p. 13), who put the case for applying existing information in what they see as a more helpful way. They argue, to summarize simply a complex argument, in favour of costing the services provided rather than hospitals or specialities, using as their indicator of efficiency the relationship between clinical salaries and the work load. The second is represented by a King's Fund working party ${ }^{2}$ who put the case for a new information system, embracing ultimately not only the hospital and the general practitioner sectors but also the local authority social services. They argue for a record-linkage system which would allow costs to be allocated to individual patients, so allowing expenditure on particular forms of treatment and on a particular patient or diagnostic groups to be worked out.

There are difficulties about both approaches. The Opit and Cross method is welcome for its suggestion that more emphasis should be put on clinical "productivity"-albeit a rather imprecise formula-as distinct from beds as the basic unit of accountancy in allocation of resources. Beds matter only in terms of the activity they generate and this in turn largely depends on the decisions of clinicians: whom to adimit, how to treat them, and when to discharge. If Opit and Cross are right in their conclusion that other hospital expenditure - on the nursing and diagnostic services-is a function of the number of doctors employed to deal with a particular case load, then clearly planning becomes both simpler and more sensible if it concentrates on improving the output of the clinicians.

But is the measure of clinical productivity proposed by Opit and Cross sufficiently accurate to become a planner's tool? Here there is doubt. Firstly there is the well known and very real problem of measuring work load. Even Hospital Activity Analysis, sophisticated though it is, does not record severity of illness, for example. ${ }^{3}$ It could be, for instance, that the Liverpool Region's performance-the lowest of the three regions examined by Opit and Cross-may be relatively even poorer than their index suggests. There is some evidence ${ }^{4}$ that hospitals in that region admit less severely-ill patients than regions with fewer beds per population. Secondly, it cannot be assumed, as Opit and Cross argue, that as any clinical service improves in quality the productivity index can be expected to fall. Such a fall would be consistent either with improving quality or a decreasing tempo of work-the two are not necessarily related. Similarly, a rise in the index could reflect either a fall in quality or the admission of less severely ill patients.

The King's Fund report shows more awareness of such problems. In particular, it emphasizes development of a 
capacity to measure the "output" of the N.H.S. in terms of measuring the outcome of treatment. Strangely, it does not put nearly as much emphasis on measuring the input, though the productivity of the N.H.S. can be assessed-if at allonly if there is some way of measuring the value-added dimension, the extent to which there has been an improvement in health. Indeed, a model for doing precisely this has been put forward by Rosser and Watts. ${ }^{5}$ Equally, it realizes that effective allocation of resources demands an information system which can compare costs of treatment in hospital with those provided by the general practitioner backed by community services. Hence the working party's proposals for moving towards a system of records which ignores the present administrative boundaries.

The obstacles to such a development, as the King's Fund report recognizes, are great. General practitioners would have to add patient identification data and medical condition or diagnosis whenever they recorded a prescription. Both the public and the profession are worried about problems of confidentiality. It would mean developing a simplified, action-orientated classification of diseases and disabilities so as to be able to relate costs of treatment to clarify defined conditions. It would also be expensive. No wonder, then, that the working party cautiously urges much preliminary investigation and a field trial. However desirable in theory such an information system may be, it will not be available to guide the allocation of N.H.S. resources within the next decade.

This may be regrettable but is not disastrous. For there is a risk that in spelling out what is a sound case for a better information system its usefulness will be over-stated. At least some of the information required to decide what forms of treatment are most cost-effective-namely, to achieve the desired result at the least cost-can be obtained by special studies. The recent study of the effects of different methods of treating varicose veins is a case in point, ${ }^{6}$ and there is still ample scope for this approach. ${ }^{7}$ Furthermore, information helps policy-makers to decide but does not replace the need for judgements based on professional or political values. For there is a temptation to think of information as a substitute for such judgements instead of as an aid in removing some of the guess-work. Even the authors of the King's Fund report do not entirely avoid this danger when they write that in allocating resources "the first step must always be to define objectives," and that the success with which these are achieved may then be "monitored through the costing system."

At the end of an era in which it has been insufficiently realized that one patient treated at too great a cost may mean another patient untreated, this emphasis is understandable. But management by objectives can all too easily come to favour those aims which can be easily quantified (and therefore monitored) at the expense of those where it is more difficult to be precise. ${ }^{8}$ What is more, the costbenefit approach could be used to justify increasing resources in those sectors of the N.H.S. where it is possible to demonstrate an economic benefit-the curing as distinct from the caring sectors. It need not do so and it should not do so. The best use must be made of scarce resources and a better information system will help towards this goal. But it is not a recipe for taking the agony out of deciding between competing priorities.

1 Department of Health and Social Security. Management Arrangements for the Reorganized National Health Service. London, H.M.S.O., 1972. Accounting for Health, Report of a King's Fund Working Party. King

${ }^{3}$ Ashley, J. S. A., British Fournal of Preventive and Social Medicine, 1972, Vol. 26, No. 3, 135-147.

${ }^{+}$Logan, R. F. L., Ashley, J. S. A., Klein, Rudolf, and Robson, D. M., Dynamics of Medical Care, Memoir No. 14. London School of Hygiene and Tropical Medicine, 1972

5 Rosser, R. M., and Watts, V. C., The Measurement of Hospital Output, International Journal of Epidemiology Vol. 1, No. 4, Winter 1972, 361-367.

${ }^{6}$ Piachaud, D., and Weddell, J. M., Proceedings of the Sixth International Scientific Meeting of the International Epidemiology Association, Belgrade, 1973, 2, 951 .

' Cochrane, A. L., Effectiveness and Efficiency. London, 1972.

${ }^{3}$ Klein, Rudolf, The Political Quarterly, 1973, 3, 270.

\section{Spread of Colds}

Coughs and sneezes spread diseases, but do they spread the common cold? A large variety of viruses may cause "colds." The commonest are rhinoviruses, ${ }^{12}$ but influenza and parainfluenza viruses, respiratory syncytial virus, adenoviruses, and coronaviruses may also do so.

Comparatively little is known about how colds are spread. Exposure of susceptible people to others with naturally or experimentally acquired colds gave low attack rates. But the intranasal inoculation of filtered rhinovirus-infected nasal washings may induce colds in from $17 \%$ to $95 \%$ of volunteers. ${ }^{3}$ Flash photography has shown that in talking droplets were excreted from the mouth but not the nose. ${ }^{4}$ From a sneeze droplets with velocity of up to $150 \mathrm{ft}(45 \mathrm{~m})$ per second may be released. 5 Again most of these droplets are from the mouth, ${ }^{6}$ though a big sneeze not trapped in a handkerchief will probably release some nasal secretions as large droplets. ${ }^{4}$ Nasal secretions are more likely to contain rhinovirus than oral secretions, and even though coughing results in droplets being released from the lower respiratory tract rhinoviruses are not common pathogens there.

Direct transmission of virus to susceptible people by large droplets from the nose is theoretically possible, but most of them fall away to the ground rapidly. While droplet nuclei may remain airborne for extended periods, to be wafted from place to place by air currents, many respiratory viruses lose much of their infectivity by drying in air, ${ }^{7}$ though the rate at which infectivity declines also depends on temperature and humidity. ${ }^{8}$

Recently J. O. Hendley and his colleagues 9 in the U.S.A. have provided some new information about the transmission of colds caused by rhinovirus. They confirmed that naturally infected patients shed virus in high titres from the nose but much less frequently and at lower titres from the throat, saliva, and cough secretions. Furthermore, when 25 naturally infected patients with common colds coughed or sneezed, only two shed virus, probably because rhinoviruses were absent from the saliva or present only in low concentrations.

Despite the poor recovery of rhinoviruses from specimens obtained from coughs and sneezes virus could be recovered from the hands of four of 10 persons with naturally acquired colds. This may have resulted from finger contact with concentrated nasal secretions during nose blowing, rubbing, or sneezing. Furthermore, in experiments in which rhinovirus-containing fluid was placed on the hands of volunteers and allowed to dry, infectious virus could be recovered up to three hours later when hands were kept still and for a slightly shorter time when hands were in use- 